\title{
Scientific Inference and the Earth's Interior: Harold Jeffreys and Dorothy Wrinch at Cambridge
}

\author{
By: Teru Miyake
}

Between 1919 and 1923, Harold Jeffreys and Dorothy Wrinch, who had both been educated at Cambridge in the 1910's, co-wrote a series of papers on a wide range of topics, from scientific inference to seismology. These papers were deeply influenced by discussions about epistemology, inference, and probability that were taking place among philosophers at Cambridge at the time. The work in these papers formed the basis for Jeffreys's later views on scientific inference and probability, and his development of seismological techniques for extracting information about the deep interior of the earth from seismic wave observations. The aim of this paper is to examine how the views of Wrinch and Jeffreys about scientific inference emerged in response to the work of the Cambridge philosophers W. E. Johnson and C. D. Broad, and then how the views of Jeffreys on inference developed in relation to the epistemological needs of the emerging field of seismology.

Harold Jeffreys is widely regarded as one of the founders of modern geophysics, a master in the application of physics to the extraction of knowledge about the interior of the earth. He and his student Keith Bullen developed the first detailed models of the interior of the earth based on observations of travel times of seismic waves. Jeffreys is also known to philosophers of probability for his objective Bayesian approach to the foundations of probability, and his debates with R. A. Fisher over frequentism. Dorothy Wrinch is not as well-known a figure as Harold Jeffreys, but her contributions to several different fields in the early twentieth century are underappreciated. Wrinch was a student of mathematics at Cambridge who later switched to philosophy after having heard Russell lecture on epistemology. She studied logic after 1916 under Russell, and spent some time as Russell's assistant. She is now most well-known for work she did after the 1930's in the application of mathematics to molecular biology, and research on protein structure in particular. Between 1919 and 1923, Wrinch and Jeffreys co-wrote a series of papers that appeared in Philosophical Magazine and Nature, in which they cover a wide range of topics, including the testing of Einstein's theory of relativity, the theory of probability, scientific inference, and seismology. They are all connected by a concern for the application of ideas about scientific inference, arising from Cambridge philosophers such as W. E. Johnson and C. D. Broad, to open scientific problems of the day. Jeffreys was clearly encouraged by the potential that these ideas had, but they could not be straightforwardly applied to the sciences in which he was most interested. His interests were in astronomy and geology, having previously written a paper on the origins of the solar system, and another on the issue of testing the theory of relativity. These are sciences where the aim is to determine facts about particular objects-e.g., what are the mechanical properties of the material $1000 \mathrm{~km}$ under the surface of the earth? On the other hand, most of the discussions about inductive inference at Cambridge centered on enumerative induction. In enumerative induction, one generalizes from particular objects of some type to particular objects of the same type, whereas the kind of inference Jeffreys wanted to do involved inferring facts about particular objects (the interior of the earth) from facts about other parts of those particular objects (the surface of the earth). Wrinch and Jeffreys thus took ideas arising in Cambridge philosophical circles and developed them so that they could be applied to the scientific problems in which they were interested.

Jeffreys developed his views in response to the epistemological needs of his own research, which increasingly centered on seismology in the 1920's and later. Seismology is the science of trying to determine facts about the deep interior of the earth, given observations of seismic waves at its surface. There are two problems that are immediately obvious when one considers the epistemology of 
seismology. First, there is a worry about underdetermination. Observations can only be done at the surface of the earth, so radically different models of the interior of the earth could potentially be consistent with all observations. Second, there is a worry about idealization. In order to extract information about the interior of the earth, one must inevitably make certain idealizations, such as isotropy of the medium in the deep interior of the earth. The views of Jeffreys about scientific inference often strike philosophers as ad hoc, but part of the reason for this is that Jeffreys develops his views in response to epistemological needs arising from scientific practice, particularly his work in geophysics and seismology. The epistemological standards of scientific practice might well diverge from those of philosophers, although it is open to question whether they ought to do so. This paper is not so much concerned with the philosophical viability of the views developed by Wrinch and Jeffreys, on which there is already an existing literature. Rather, we have here an interesting case where a scientist takes ideas that originally arose in philosophical debates, and attempts to apply them in actual scientific practice, and then these ideas are further developed in response to the needs of scientific practice. This paper thus focuses on the following questions: What did Jeffreys think was needed for a theory of inference and probability that would provide a sufficient foundation for his work in geophysics, and seismology in particular? How did the needs of geophysical research influence the way that Jeffreys thought about scientific inference? How, on the other hand, did ideas arising from philosophical discussions help Jeffreys to develop the methods of seismology? 